\title{
Evaluation of the serum zinc level in patients with vitiligo
}

\author{
Majid Rostami Mogaddam¹, Nastaran Safavi Ardabili², Nasrollah Maleki ${ }^{3}$, Mir Mehdi Chinifroush ${ }^{4}$, \\ Elham Maleki Fard ${ }^{1}$
}

\begin{abstract}
${ }^{1}$ Department of Dermatology, Imam Khomeini Hospital, School of Medicine, Ardabil University of Medical Sciences, Ardabil, Iran ${ }^{2}$ Department of Midwifery, Ardabil Branch, Islamic Azad University, Ardabil, Iran

${ }^{3}$ The Persian Gulf Tropical Medicine Research Center, Department of Internal Medicine, Bushehr University of Medical Sciences, Bushehr, Iran

${ }^{4}$ Department of Pathology, Imam Khomeini Hospital, School of Medicine, Ardabil University of Medical Sciences, Ardabil, Iran
\end{abstract}

Adv Dermatol Allergol 2017; XXXIV (2): 116-119

DOI: https://doi.org/10.5114/ada.2017.67073

\begin{abstract}
Introduction: Vitiligo is an acquired, idiopathic disorder characterized by circumscribed depigmented macules and patches, which affects approximately $0.1-2 \%$ of the general population worldwide. Zinc is an essential trace element that is necessary for growth and development at all stages of life. Some studies have reported an association between serum zinc levels and vitiligo.

Aim: To measure the serum zinc level in patients with vitiligo compared to healthy subjects.

Material and methods: One hundred patients with vitiligo and 100 healthy controls were referred to our clinic. The two groups were matched for age and sex. Atomic absorption spectrophotometry was used to measure serum zinc levels. The statistical analysis was performed using SPSS software.

Results: The mean serum level of zinc in vitiligo patients and controls was $80.11 \pm 17.10 \mu \mathrm{g} / \mathrm{dl}$ and $96.10 \pm 16.16 \mu \mathrm{g} / \mathrm{dl}$, respectively. The serum zinc level in patients with vitiligo was significantly lower than in healthy controls $(p=0.0001)$.

Conclusions: The results of our study revealed a significant association between vitiligo and serum zinc levels. A relative decrease in the serum zinc level in vitiligo patients can highlight the role of zinc in the pathogenesis of vitiligo, and large-scale studies need to be conducted to confirm these findings and assess the effect of oral zinc supplements in patients with low zinc levels.
\end{abstract}

Key words: vitiligo, serum level, zinc.

\section{Introduction}

Vitiligo is an acquired, idiopathic disorder characterized by circumscribed depigmented macules and patches, which affects approximately $0.1-2 \%$ of the general population worldwide [1, 2] and it may appear any time from shortly after birth to senescence. The average age of onset is variable, but peaks in the second and third decades [3]. The exact pathogenesis of vitiligo is uncertain [4]. Vitiligo is a multifactorial disorder related to both genetic and non-genetic factors [5, 6]. It is generally agreed that there is an absence of functional melanocytes in the vitiligo skin and that this loss of histochemically recognizable melanocytes is a result of their destruction [4, $7,8]$. There are three basic techniques of genetic studies: candidate gene association studies, genome-wide linkage studies and genome-wide association studies (GWAS) [9].

The diagnosis of vitiligo is based upon the clinical presence of depigmented patches of the skin. Examination with a Wood's lamp is useful for highlighting areas of pigment loss in lightly-pigmented patients. A full body skin examination should be performed in adult patients with new-onset vitiligo to exclude a melanoma-associated depigmentation. Increased frequencies of other autoimmune disorders, such as autoimmune thyroid disease, pernicious anemia, systemic lupus erythematosus, and Addison disease have been detected in patients with vitiligo $[10,11]$. The association of vitiligo with these disorders suggests the presence of shared genetic factors that contribute to the development of these diseases.

Address for correspondence: Dr. Nasrollah Maleki, The Persian Gulf Tropical Medicine Research Center, Department of Internal Medicine, Bushehr University of Medical Sciences, Bushehr, IR Iran, zip code: 7514763448, phone: +989163057715, e-mail: malekinasrollah@yahoo.com Received: 21.01.2015, accepted: 3.03.2016. 
Zinc is an essential trace element that is necessary for growth and development at all stages of life [12]. Zinc plays a key role in physical growth and development, functioning of the immune system, reproductive health, sensory function and neurobehavioral development. It has been estimated that around 33\% of the world's human population have diets deficient in zinc, but this ranges between $4 \%$ and $73 \%$ in different countries [13]. Mild zinc deficiency is associated with depressed immunity, impaired taste and smell, onset of night blindness, and decreased spermatogenesis. Severe zinc deficiency is characterized by severely depressed immune function, frequent infections, diarrhea, and alopecia [14].

Some investigators have reported an association between low serum zinc levels and vitiligo, while others have not found the same.

\section{Aim}

This study was conducted to measure the serum zinc level in patients with vitiligo and compare it with healthy controls.

\section{Material and methods}

\section{Patients and controls}

This study was approved by the Ethics Committee of the Ardabil University of Medical Sciences. This was a prospective cross-sectional study. Patients presenting with vitiligo to the dermatology outpatient clinic of Imam Khomeini Hospital in Ardabil, Iran, between March 2012 and April 2013 were recruited for the study. A total of 100 patients with acne vulgaris and 100 healthy controls were included. The two groups were matched for age and sex. The diagnosis of vitiligo was based upon the clinical presence of depigmented patches of the skin and examination with a Wood's lamp.

The inclusion criteria for patients with vitiligo were: being over 15 years of age, being at least a primary school graduate, not taking any medication for any purposes, and being willing to participate in the study. Patients were excluded if they had any disfiguring facial condition other than vitiligo, history of active malignancy, under immunosuppressive treatment, liver cirrhosis, renal failure, under any treatment with zinc in the 1 month prior to diagnosis, pregnancy, alcoholism, malabsorption disorders, physical disability, any neurological disorder, or other physical diseases, which might cause psychological distress.

A questionnaire was completed for each patient, which included the data of demographic status, medical and drug history, duration of vitiligo, and familial status for vitiligo. Then, for all participants, laboratory tests were recommended, which comprised complete blood count, serum calcium, phosphorus and zinc levels, fasting blood sugar, liver function, renal function and thyroid function tests, urinalysis and stool examination. Written informed consent was obtained from the participants.

\section{Measurement of the serum zinc level}

A $3 \mathrm{ml}$ intravenous blood sample was taken from eligible cases and controls, and then taken in a heparinized and sterile test tube at $-40^{\circ} \mathrm{C}$. Blood samples were withdrawn by zinc-free plastic syringes and placed in zinc-free centrifuge tubes. Atomic absorption spectrophotometry (Varian Spectra AA-10 Model) was used to measure serum zinc levels. The normal value of the serum zinc level in adults was accepted as 70-140 $\mu \mathrm{g} / \mathrm{dl}$.

\section{Statistical analysis}

The statistical analysis of the data was done using SPSS software (Version 19.SPSS Inc, United States). The comparison of the continuous variables was accomplished with the Student's t test, and for the comparison of the categorical variables, the $\chi^{2}$ test was used. The Pearson correlation analysis was used for the evaluation of the correlation between scores of the different scales and other relevant variables. A $p$-value of $<0.05$ was considered statistically significant. The results were expressed as means \pm standard deviations.

\section{Results}

A total of 100 vitiligo patients and 100 healthy volunteers were enrolled in this study. Each group included 54 (54\%) men and 46 (46\%) women. The mean age of the patients was $24.97 \pm 2.58$ years, and the mean age of the controls was $25.32 \pm 2.47$ years. The two groups showed no significant differences in age or gender. The highest prevalence was in the age range of 20 to 29 years (34\%).

The mean duration of the disease was $41.37 \pm 26.41$ months, with a range between 6 and 120 months. Only $17 \%$ of patients had a positive family history. In the vitiligo group, 79 (79\%) patients were single and 21 (21\%) patients were married. The mean duration of education in this group was $12.39 \pm 2.85$ years. In the control group, 81 (81\%) patients were single and 19 (19\%) patients were married. The mean duration of education in this group was $11.97 \pm 2.81$ years. None of the above demographic variables showed any statistically significant differences between the vitiligo group and the control group. The most common type of vitiligo was the generalized type (81\%) followed by segmental vitiligo (10\%) and focal vitiligo (9\%). The most common site of involvement was found in the lower extremities.

The average serum levels of zinc in vitiligo patients and controls are presented in Table 1 . The serum zinc level in controls ranged from 65 to $120 \mu \mathrm{g} / \mathrm{dl}$ with a mean value of $96.10 \pm 16.16 \mu \mathrm{g} / \mathrm{dl}$. The serum zinc level in vitiligo patients ranged from 30.10 to $120 \mu \mathrm{g} / \mathrm{dl}$, with a mean value of $80.11 \pm 17.10 \mu \mathrm{g} / \mathrm{dl}$ (Table 1 ). Paired $t$-test results showed that there is a significant difference between the zinc serum level in patients with vitiligo compared with healthy subjects $(p=0.0001)$. There was no significant 
Table 1. The mean serum zinc level in vitiligo patients and controls

\begin{tabular}{lccc}
\hline Study subjects & \multicolumn{2}{c}{ Serum zinc $[\mu \mathrm{g} / \mathrm{dl}]$} & \multirow{2}{*}{$P$-value } \\
\cline { 2 - 3 } & Range & Mean \pm SD & \\
\hline Vitiligo group & $30.1-120$ & 80.11 & 0.0001 \\
$(N=100)$ & & \pm 17.10 & \\
\hline Control group & $65-120$ & 96.10 & \\
$(N=100)$ & & \pm 16.16 & \\
\hline
\end{tabular}

correlation between the serum level of zinc with a family history of the disease $(p=0.19)$, type of vitiligo $(p=0.25)$, and duration of disease $(p=0.25)$.

Mean serum levels of calcium, phosphorus, fasting blood sugar (FBS), and thyroid stimulating hormone (TSH) in vitiligo patients and controls are presented in Table 2. None of mean serum levels of calcium, phosphorus, FBS showed any statistically significant differences between the vitiligo group and the control group. Among patients with vitiligo, (18) $18 \%$ had thyroid dysfunction, while in the control group, 9 (9\%) had thyroid dysfunction. There was a significantly higher prevalence of thyroid dysfunction in patients with vitiligo compared with the control group $(p=0.002)$. However, there was no significant difference in the mean levels of TSH between the patient and control group $(p=0.104)$.

\section{Discussion}

Vitiligo is an acquired pigmentary disorder of the skin and mucous membranes and is characterized by circumscribed, depigmented macules and patches. It commonly begins in childhood or young adulthood [15]. The cosmetic impact of this disease is tremendous and its psychological impact is devastating, particularly in colored races [16].

Theories regarding destruction of melanocytes of vitiligo include autoimmune mechanisms, cytotoxic mechanisms, intrinsic melanocyte defects, oxidant-antioxidant mechanisms, and neural mechanisms. The autoimmune theory proposes an alteration in humoral and cellular immunity in the destruction of melanocytes of vitiligo. Autoimmune thyroid diseases, particularly Hashimoto thyroiditis and Graves disease; other endocrinopathies, such as Addison disease and diabetes mellitus; and alopecia areata; pernicious anemia; inflammatory bowel disease; psoriasis; and autoimmune polyglandular syndromes are all associated with vitiligo. This indicates the presence of genetically determined susceptibility to not only vitiligo but also to other autoimmune disorders [17]. Oxidant stress may also play an essential role in the pathogenesis of vitiligo. Studies suggest that accumulation of free radicals toxic to melanocytes leads to their destruction [18].

Although the diagnosis of vitiligo generally is made on the basis of clinical findings, biopsy is occasionally helpful to differentiate vitiligo from other hypopigmentary disorders. Two scores designed for the assessment of vitiligo are the vitiligo area severity index (VASI) and Vitiligo European Task Force (VETF) [19].

Zinc is a potential antiapoptotic factor. On the other hand, based on histological and some laboratory data, apoptosis of melanocytes has been suggested as a probable mechanism of vitiligo. Hence, zinc, via preventing apoptosis of melanocytes may be able to control vitiligo [20]. Zinc in combination with other micronutrients plays an important role in the process of melanogenesis. They catalyze the rearrangement of dopachrome to from 5, 6-dihydroxy indole-2-carboxylic acid, and enhancement of eumelanin polymer formation from monomers. This process is the final stage of eumelanin formation in melanogenesis so zinc may have an important effect on vitiligo [21, 22].

A number of studies have been made to reveal the effect of zinc on vitiligo. The present study showed significantly lower levels of zinc in serum of patients with vitiligo compared to the control group ( $p=0.0001)$. Molokhia and Portnoy [23] conducted a study to estimate copper and zinc levels in serum and skin of vitiligo patients; they found a significant reduction in serum zinc, while the results of copper analysis in vitiligo neither support a deficiency role for copper nor justify its use in the treatment of that disease. Tasaki et al. [24] conducted a study to estimate serum copper (Cu) and zinc (Zn) levels and copper/zinc ratios in 151 cases of various skin diseases. The serum level of Zn was significantly decreased in cases of bullous pemphigoid, decubitus ulcer, and alopecia areata. The serum level of Cu was elevated in cases of psoriasis, decubitus ulcer, and skin cancer. They also demonstrated that, in each skin disease, the Cu/Zn ratio clearly reflects the severity of the progress.

Table 2. The mean serum levels of calcium, phosphorus, fasting blood sugar (FBS), and thyroid stimulating hormone (TSH) in vitiligo patients and controls

\begin{tabular}{lccc}
\hline Serum level & Vitiligo group $(N=100)$ & Control group $(N=100)$ & $P$-value \\
\hline Calcium $[\mathrm{mg} / \mathrm{dl}]$ & $9.3 \pm 0.49$ & $9.4 \pm 0.41$ & 0.826 \\
\hline Phosphorus $[\mathrm{mg} / \mathrm{dl}]$ & $3.2 \pm 0.1$ & $3.4 \pm 0.2$ & 0.875 \\
\hline FBS $[\mu \mathrm{lU} / \mathrm{ml}]$ & $86.49 \pm 11.21$ & $89.68 \pm 12.48$ & 0.373 \\
\hline TSH $[\mu \mathrm{lU} / \mathrm{ml}]$ & $2.94 \pm 4.86$ & $3.19 \pm 4.69$ & 0.104 \\
\hline
\end{tabular}


Zinc- $\alpha 2$-glycoprotein (ZAG) is a recently identified adipokine, assigned to chromosome 7q22.1. The ZAG plays a role in lipolysis, regulation of metabolism, cell proliferation and differentiation, regulation of melanin synthesis, cell adhesion, immunoregulation, and so forth [25]. Zinc via precipitating the ZAG in site of vitiligo patches may be effective in the treatment of vitiligo $[25,26]$. Some studies showed a significant increase in the percentage of apoptosis in peripheral blood mononuclear cells in vitiligo. On the other hand, the accumulation of toxic compounds, altered cellular environment and infection can all contribute to vitiligo. Zinc may have an effect on preventing vitiligo via destructing these probable environmental factors through prevention of these immunityrelated cells [20].

In 2014, Zeng et al. [27] conducted a meta-analysis to compare the serum levels of $\mathrm{Cu}$ and $\mathrm{Zn}$ between vitiligo patients and healthy controls. In this meta-analysis, 16 studies with a total of 891 vitiligo cases and 1682 healthy controls were conducted. The serum levels of Cu were significantly lower in vitiligo patients than in healthy controls $(p<0.0001)$. The levels of serum $\mathrm{Zn}$ were also significantly lower in vitiligo patients than in healthy controls $(p<0.00001)$. These results demonstrated that decreased levels of serum $\mathrm{Cu}$ and $\mathrm{Zn}$ are generally present in vitiligo patients [27].

Further investigations with a larger number of patients and also dividing them into further classifications of these diseases to obtain better knowledge of the effect of zinc are needed. Also, treatment with zinc supplements can be tried in these patients to see the outcome.

\section{Conclusions}

The results of our study revealed a significant association between vitiligo and serum zinc levels. A relative decrease in the serum zinc level in vitiligo patients can highlight the role of zinc in the pathogenesis of vitiligo, and large-scale studies need to be conducted to confirm these findings and assess the effect of oral zinc supplements in patients with low zinc levels.

\section{Conflict of interest}

The authors declare no conflict of interest.

\section{References}

1. Howitz J, Brodthagen H, Schwartz M, Thomsen K. Prevalence of vitiligo: epidemiological survey on the Isle of Bornholm, Denmark. Arch Dermatol 1977; 113: 47-52.

2. Alkhateeb A, Fain PR, Thody A, et al. Epidemiology of vitiligo and associated autoimmune diseases in Caucasian probands and their families. Pigment Cell Res 2003; 16: 208-14.

3. Handa S, Kaur I. Vitiligo: clinical findings in 1436 patients. J Dermatol 1999; 26: 653-7.

4. Misterska M, Szulczyńska-Gabor J, Żaba R. Aetiopathogenesis, clinical picture and treatment of vitiligo. Postep Derm Alergol 2009; 26: 212-23.
5. Karagün E, Ergin C, Baysak S, et al. The role of serum vitamin D levels in vitiligo. Adv Dermatol Allergol 2016; 33 : 300-2.

6. Kartal D, Borlu M, Çınar SL, et al. Thyroid abnormalities in paediatric patients with vitiligo: retrospective study. Adv Dermatol Allergol 2016; 33: 232-4.

7. Malhotra N, Dytoc M. The pathogenesis of vitiligo. J Cutan Med Surg 2013; 17: 153-72.

8. Njoo MD, Westerhof W. Vitiligo. Pathogenesis and treatment. Am J Clin Dermatol 2001; 2: 167-81.

9. Czajkowski R, Męcińska-Jundziłł K. Current aspects of vitiligo genetics. Postep Derm Alergol 2014; 31: 247-55.

10. Vrijman C, Kroon MW, Limpens J, et al. The prevalence of thyroid disease in patients with vitiligo: a systematic review. Br J Dermatol 2012; 167: 1224-35.

11. Terpiłowska S, Siwicki AK. The role of selected microelements: selenium, zinc, chromium and iron in immune system. Centr Eur J Immunol 2011; 36: 303-7.

12. Kamer B, Wąsowicz W, Pyziak K, et al. Role of selenium and zinc in the pathogenesis of food allergy in infants and young children. Arch Med Sci 2012; 8: 1083-8.

13. Hotz C, Brown KH. Assessment of the risk of zinc deficiency in populations and options for its control. Food and Nutrition Bulletin 2004; 25 (1 Suppl. 2): S91-203.

14. Prasad AS. Zinc and immunity. Mol Cell Biochem 1998; 188: 63-9.

15. Klaus S, Lerner AB. Vitiligo. J Am Acad Dermatol 1984; 11: 997-1000.

16. Hautmann G, Panconesi E. Vitiligo: a psychologically influenced and influencing disease. Clin Dermatol 1997; 15: 875-8.

17. Oiso N, Suzuki T, Fukai K, et al. Nonsegmental vitiligo and autoimmune mechanism. Dermatol Res Pract 2011; 2011: 518090.

18. Passi S, Grandinetti M, Maggio F, et al. Epidermal oxidative stress in vitiligo. Pigment Cell Res 1998; 11: 81-5.

19. Kawakami T, Hashimoto T. Disease severity indexes and treatment evaluation criteria in vitiligo. Dermatol Res Pract 2011; 2011: 750342.

20. Bagherani N, Yaghoobi R, Omidian M. Hypothesis: zinc can be effective in treatment of vitiligo. Indian J Dermatol 2011; 56: 480-4.

21. Shameer P, Prasad PV, Kaviarasan PK. Serum zinc level in vitiligo: a case control study. Indian J Dermatol Venereol Leprol 2005; 71: 206-7.

22. Inamadar AC, Palit A. Acrodermatitis enteropathica with depigmented skin lesions simulating vitiligo. Pediatr Dermatol 2007; 24: 668-9.

23. Molokhia MM, Portnoy B. Neutron activation analysis of trace elements in skin. VII. Copper and zinc in vitiligo, moles and seborrhoeic warts. Br J Dermatol 1973; 88: 347-53.

24. Tasaki M, Hanada K, Hashimoto I. Analyses of serum copper and zinc levels and copper/zinc ratios in skin diseases. J Dermatol 1993; 20: 21-4.

25. Bagherani N. The newest hypothesis about vitiligo: most of the suggested pathogeneses of vitiligo can be attributed to lack of one factor, zinc-alpha2-glycoprotein. ISRN Dermatol 2012; 2012: 405268.

26. Yaghoobi R, Omidian M, Bagherani N. Vitiligo: a review of the published work 2011; 38: 419-31. J Dermatol 2011; 38: 419-31.

27. Zeng Q, Yin J, Fan F, et al. Decreased copper and zinc in sera of Chinese vitiligo patients: a meta-analysis. J Dermatol 2014; 41: 245-51. 